of Medicine and Public Health, Flinders University, SA, Australia; and ${ }^{4}$ Clinical Immunology, Flinders Medical Centre, SA, Australia

Introduction: The gold standard for diagnosing SARS-CoV-2 infection remains RT-PCR; however serological testing has an important role in epidemiology and assessment of vaccine responses. We expressed the receptor binding domain (RBD) of the spike (S) protein as a glycosylphosphatidylinositol (GPI)anchored protein on $\mathrm{CHO}$ cells transfected with a generated construct and determined binding of RBD specific antibodies by immunofluorescence (IF) and flow cytometry (FACS) assays.

Methods and results: IF and FACS demonstrated cell surface RBD expression using anti-S RBD $\mathrm{mAb}$ and macaque immune sera. GPI anchored expression was confirmed through loss of positive cells on FACS post GPI anchor cleavage with phosphoinositide phospholipase C (PI-PLC). Binding of antibodies from COVID-19 convalescent sera to RBD- and mock-transfectants was performed by FACS. Positive results on serial dilutions of patient sera down to 1:3200 were observed and compared to in-house RBD ELISA which lost positivity at a 1:800 dilution. Soluble recombinant ACE2 bound to RBDCHOs and binding was inhibited by COVID-19 convalescent sera indicating the RBD was expressed in a functional conformation and that neutralising antibodies were detected.

Conclusion: Mammalian cells expressing GPI-anchored RBD can successfully be used to detect previous SARS-CoV-2 infection and likely indicate the presence of neutralising antibodies in COVID-19 survivors.

\section{IMPACT OF A DIAGNOSTIC STEWARDSHIP INITIATIVE OF BACTERIAL 16s rRNA SEQUENCING REFERRALS FROM A REGIONAL LABORATORY}

Daniel Lennon, Rob George, Hemalatha Varadhan

New South Wales Health Pathology - Hunter, John Hunter

Hospital, New Lambton Heights, NSW, Australia

Introduction: 16s rRNA PCR (16s) has emerged as a useful tool for the identification of bacterial pathogens in clinical samples1. Due to its increased cost and limited availability, this technology is also a suitable target for diagnostic stewardship initiatives.

Aim: To assess a diagnostic stewardship initiative impact on key laboratory quality indicators for specimen referrals for $16 \mathrm{~s}$ PCR from the John Hunter Hospital Laboratory.

Methods: From 01/07/2019 to 31/12/2019, all referred specimens for 16 s received by our laboratory were held for review by a Microbiologist (or Registrar). Key laboratory quality indicators were collected and compared to a retrospective audit of $16 \mathrm{~s}$ referrals from the preceding 6 months.

Results: 192 referrals were examined, with 77 referrals made in the pre-intervention phase and 115 in the post-intervention phase. 9 referrals were cancelled in the post-intervention phase. The cost per positive 16 s was $\$ 580.04$ in the pre-intervention period and $\$ 375.40$ in the post intervention period. The average turn-around time was 14.61 days in the pre-intervention period, and 13.47 days in the post-intervention period, with no significant difference between the two periods $(p=0.122)$.

Conclusion: Diagnostic stewardship is an economical endeavor for laboratories without increase in turnaround time.

\section{Reference}

1. Janda JM, Abbott SL. 16S rRNA gene sequencing for bacterial identification in the diagnostic laboratory: pluses, perils, and pitfalls. J Clin Microbiol 2007; 45: 2761-4.

\section{FUNGAL LIVER ABSCESSES IN AN IMMUNOCOMPETENT PATIENT POST CHOLECYSTECTOMY - A CASE REPORT}

\section{Laura M. Luttrell}

Department of General Surgery, Bunbury Regional Hospital, Bunbury, WA, Australia

Candida albicans is known to colonise the biliary tract but rarely causes invasive candidiasis post gastrointestinal or hepatobiliary surgery in the absence of host immunosuppression. This case study reports a 33-year-old immunocompetent female with a past surgical history of sleeve gastrectomy, omega loop (mini) gastric bypass and gastric ulcer repair. Two weeks post laparoscopic cholecystectomy and intraoperative cholangiogram (IOC) the patient presented with right upper quadrant abdominal pain, rigors, nausea, vomiting, fevers $(39.5$ degrees) and tachycardia. Liver function tests were mildly deranged, the white cell count was $10 \times 10^{9} / \mathrm{L}$ and the C-reactive protein was elevated at $327 \mathrm{mg} / \mathrm{L}$. Computed tomography (CT) revealed multiple right lobe liver abscesses and mild splenomegaly. The patient underwent ultrasound guided drainage and Candida albicans was subsequently grown from the aspirate sample. After a 4-week course of oral fluconazole, a repeat CT abdomen and pelvis showed good resolution with 3-4 remaining small liver collections. An abdominal ultrasound 8-weeks post initial presentation showed complete resolution and subsequent gastroscope, echocardiogram and ophthalmological examination were unremarkable. Although liver abscesses caused by Candida albicans rarely occur in immunocompetent patients, it should be included in the differential diagnosis in such patients presenting with suspicious features post laparoscopic cholecystectomy and IOC.

\section{OSTEOMYELITIS CAUSED BY AGGREGATIBACTER APHROPHILUS - A CASE REPORT}

Abhinav Rajkumar $^{1}$, Gayana P. S. Gunaratna ${ }^{1,2}$,

$\overline{\text { Kristine Macartney }}^{1}$, Alexander C. Outhred ${ }^{1}$, Alison Kesson

${ }^{1}$ Department of Infectious Diseases and Microbiology, The Children's Hospital, Westmead, NSW, Australia; and ${ }^{2}$ Faculty of Medicine, University of Kelaniya, Sri Lanka

Osteomyelitis in children is often secondary to haematogenous dissemination of bacteria into long bone metaphyses. Organisms causing osteomyelitis vary with age, and the virulence of different pathogens influences clinical presentation. ${ }^{1}$ Aetiological diagnosis is important in determining appropriate antibiotic therapy.

This case involves an 11-year-old girl with 4 months worsening left knee pain and difficulty weight bearing. She had no fever, preceding trauma or recent dental procedures. Inflammatory markers were unremarkable, with radiological evidence of a Brodie's abscess. She was trialled on oral cephalexin; despite transient clinical improvement, she re-presented with worsening symptoms. 